\title{
SEM-ANALYSIS OF FRACTURE FEATURES FORMED IN EXCIMER-LASER INDUCED SURFACE DAMAGE $\mathrm{OF} \mathrm{CaF}_{2}$
}

\author{
H. JOHANSEN, ${ }^{*}$ S. GOGOLL, ${ }^{+}$E. STENZEL, ${ }^{+}$M. REICHLING, ${ }^{+}$ \\ and E. MATTHIAS ${ }^{+}$ \\ ${ }^{*}$ Max-Planck-Institut für Mikrostrukturphysik Halle, Weinberg 2, 06120 Halle, \\ Germany; ${ }^{+}$Fachbereich Physik, Freie Universität Berlin, Arnimallee 14, 14195 Berlin, \\ Germany
}

The fracture damage of bulk (111)- $\mathrm{CaF}_{2}$ crystals induced by pulsed irradiation in single shot mode with $248 \mathrm{~nm}$ laser light in air is investigated. The irradiated spots are studied with a scanning electron microscope (SEM) using different operational modes. An analysis of the fracture features yields evidence for defect induced absorption, thermal stress and shock wave generation as the main cause of damage. Fracturing is preferentially aligned along the (111)-directions resulting in a triangular shape for the tile fragments. The thickness of the fragments shows a clear dependence on incident laser fluence, what can be explained assuming an enhanced surface absorption in the $\mathrm{CaF}_{2}$ crystal.

Keywords: Laser-induced damage, alkaline-earth halides, absorption centres, energy transfer, scanning electron microscopy.

\section{INTRODUCTION}

Laser damage of bulk crystals of $\mathrm{CaF}_{2}$ can be characterized by a clear threshold behaviour ${ }^{1}$ and the dominant damage feature is cracking along the natural cleavage planes. ${ }^{2}$ To uncover thermomechanical details of the damage process one needs topographical information about the microscopic surface modification of the crystal and of the irradiation-induced fragments. With respect to the latter conventional SEM is especially appropriate, where a full depth of field but only a limited lateral resolution in the range of about $20 \mathrm{~nm}$ is required. Furthermore, to clearly interpret such phenomena as detachment of crystallites from the bulk, local melting, or the formation of droplets, it is necessary to obtain the specific contrast information of secondary electrons (SE) and backscattered electrons $(\mathrm{BE})$ related to possible phase transitions in local areas of the laser irradiation.

A careful analysis of topographic damage effects by means of SEM reveals traces of the interaction process in different depths of the crystal, for example, on the surface of tiles or craters, on the subsurface below the tiles, and in form of locally detectable absorption centres in the depth. The lateral temperature variation at the periphery due to the average temperature increase in the centre of the spot entails fracture as the most significant feature. From the multitude of information revealed by an inspection of the damaged area we have choosen one topic for this contribution, namely the relationship between the fluence of single laser pulses and the resulting thickness of the detached tiles.

\section{EXPERIMENTAL}

The damage behaviour was measured in a 1-on-1 shot irradiation mode. An excimer laser provided $14 \mathrm{~ns}$ pulses of $248 \mathrm{~nm}$ light. The laser beam was guided through an aperture and 


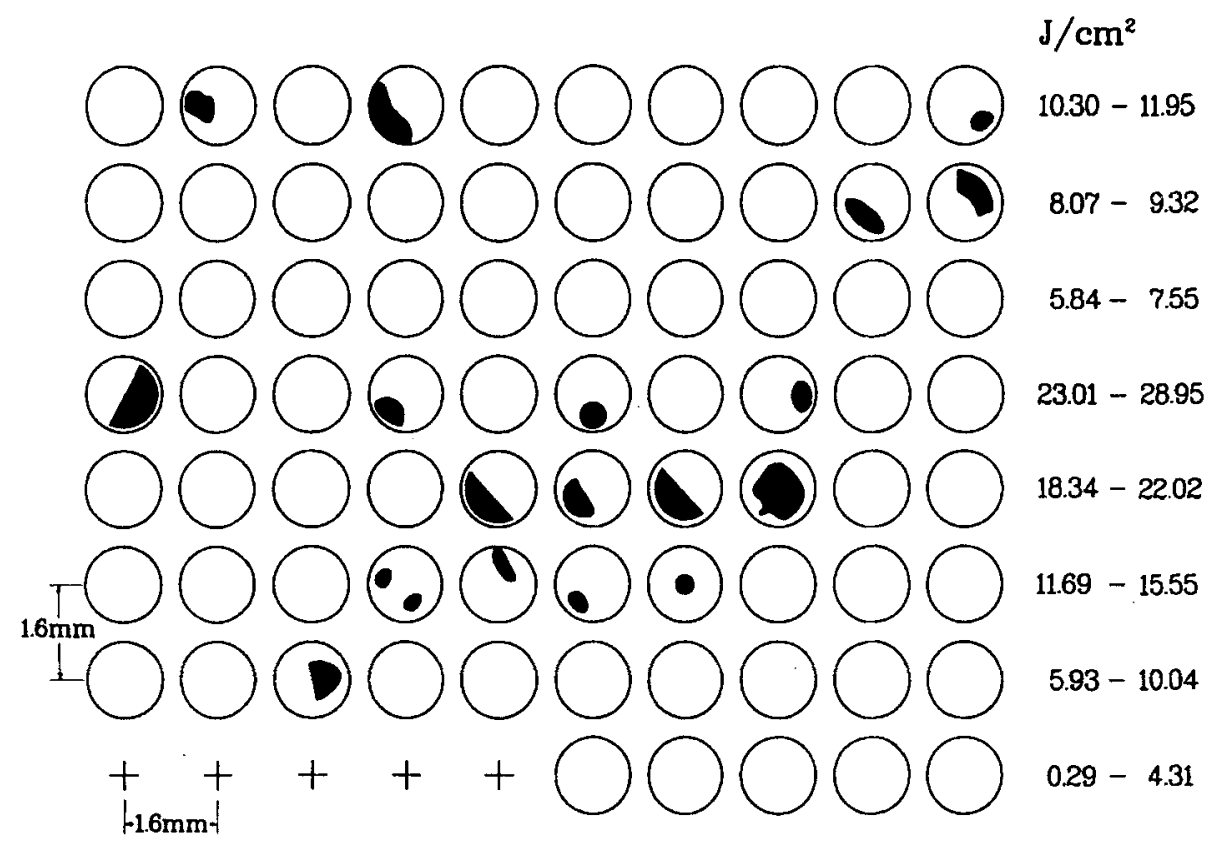

FIGURE 1(a) Distribution of extended defect regions on the crystal surface after 1-on-1 shot irradiation with $248 \mathrm{~nm}$ laser light. Crosses: no damage, open circles: normal damage (tile detachment) marked regions in circles: indication for degree and morphology of massive damage in defect rich regions.

imaging optics providing a close to top-hat profile at the crystal surface. The length of the principal axes of the eleiptically shaped spot was $110 \mu \mathrm{m}$ and $175 \mu \mathrm{m}$, respectively. Pulse energy was varied in the range of 0.3 to $30 \mathrm{~mJ} /$ pulse. In addition to the incident light the transmitted light intensity was monitored. ${ }^{3}$ Due to the short focal length of the imaging optics the intensity on the rear face of the crystal was too low to cause backside damage.

The experiments were performed in air with an optically polished UV-grade (111) $\mathrm{CaF}_{2}$ single crystal $\left(20 \times 20 \times 6 \mathrm{~mm}^{3}\right)$ purchased from Karl Korth company, Kiel.

To ensure SEM surface investigations free of charging, the crystal surface was covered with a conducting $15 \mathrm{~nm}$ carbon layer prior to the SEM measurements. The SEM-BE composition mode was found to be most suitable for a sensitive investigation of microcracks as low density regions, whereas potential contrast arises due to the positive charge-up of tiles using the SE mode at primary energies below $1 \mathrm{keV}$.

\section{RESULTS AND DISCUSSION}

Figure 1(a) displays a schematic survey over the damage results. As indicated by the numbers given on the right hand side, the single shot irradiation on the (111)-surface of $\mathrm{CaF}_{2}$ was carried out in series with increasing intensity ordered in horizontal lines. Irradiated locations without any detectable damage are marked by crosses, the others by circles. The typical damage morphology of most of the damage spots is shown in Figure 1(b) and can be characterized by a detachment of $\mathrm{CaF}_{2}$ tiles. The fluence varies 


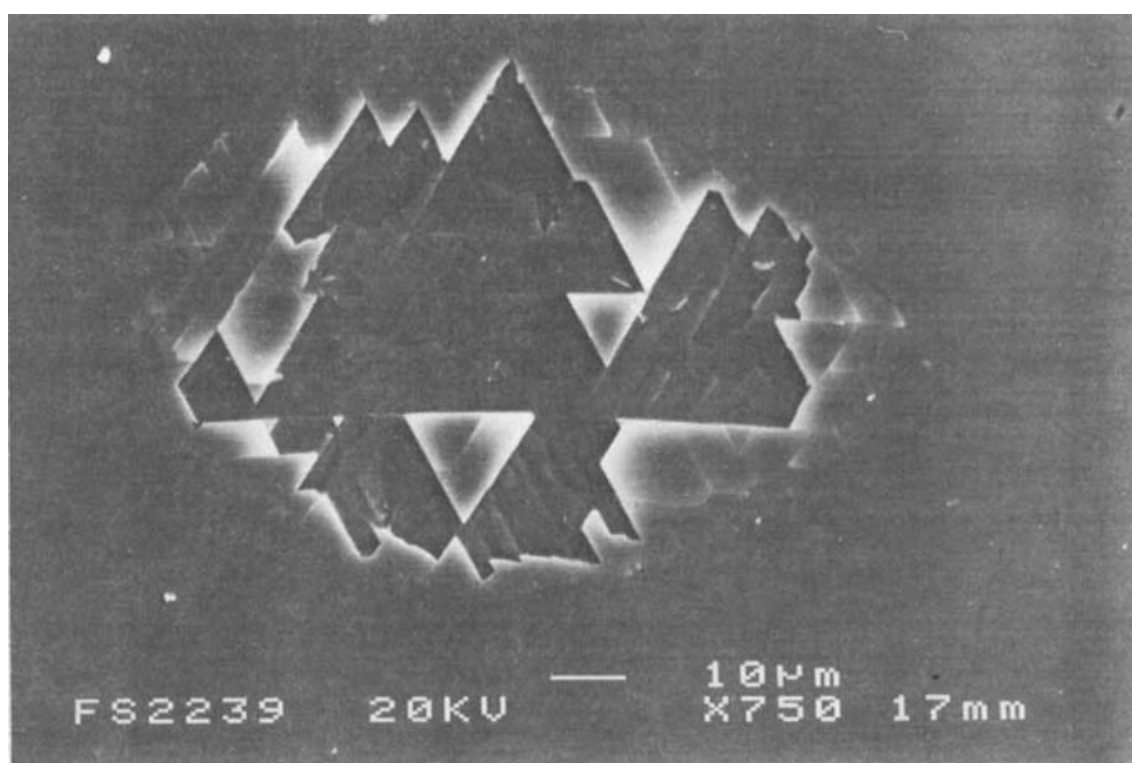

FIGURE 1(b) SE micrograph illustrating 'normal' damage at $13.5 \mathrm{~J} / \mathrm{cm}^{2}$.

from 0.3 to $30 \mathrm{~J} / \mathrm{cm}^{2}$ in different ranges in each of these series. Although, a damage threshold can be determined (about $4 \mathrm{~J} / \mathrm{cm}^{2}$ ), there is no clear correlation between the extent of damage and laser fluence. Systematic SEM inspection reveals that at some irradiated spots a pronounced three-dimensional ejection of material had taken place. A typical example is shown in Figure 1(c) where a damage depth much larger than the average tile thickness is found. In Figure 1(a) those spots are represented as circles with marked regions inside, where the degree and morphology of the damage is indicated by the shading. This nonuniform distribution of severe damage characterizes the unequal quality of the original crystal and/or the influence of the polishing procedure. Local defect aggregates of unknown nature may be the source of this phenomenon. The position of the strong damage area within each spot clearly varies between different areas and does not result from inhomogeneities of the laser intensity profile. After careful inspection of the whole crystal complete series without such strong features could be found.

Investigating the damage behaviour in detail outside the strong damage regions represented in Figure 1, SEM examination reveals an onset of coherent planar damage at a pulse energy fluence of about $4 \mathrm{~J} / \mathrm{cm}^{2}$. This is coincident with the observed decrease of the optical transmission. ${ }^{3}$ Fine surface cracks having no conjunction among each other are detectable with sufficient contrast down to $\approx 2.6 \mathrm{~J} / \mathrm{cm}^{2}$ by the SEM-BE composition mode. Fracture mostly occurs along the natural (111)-cleavage planes. Since there are four different (111)-planes intersecting the surface, rectangular patterns are expected. This is found for $\mathrm{CaF}_{2}(100)$. On the (111)-surface, however, the three remaining (111)-planes form tiles in the shape of equilateral triangles, trapezoids and parallelograms as found in Figure 2. Here, a local detachment of tiles vertical and parallel to the surface can be detected sensitively by the electron beam induced voltage contrast (EBIVC) for a primary 


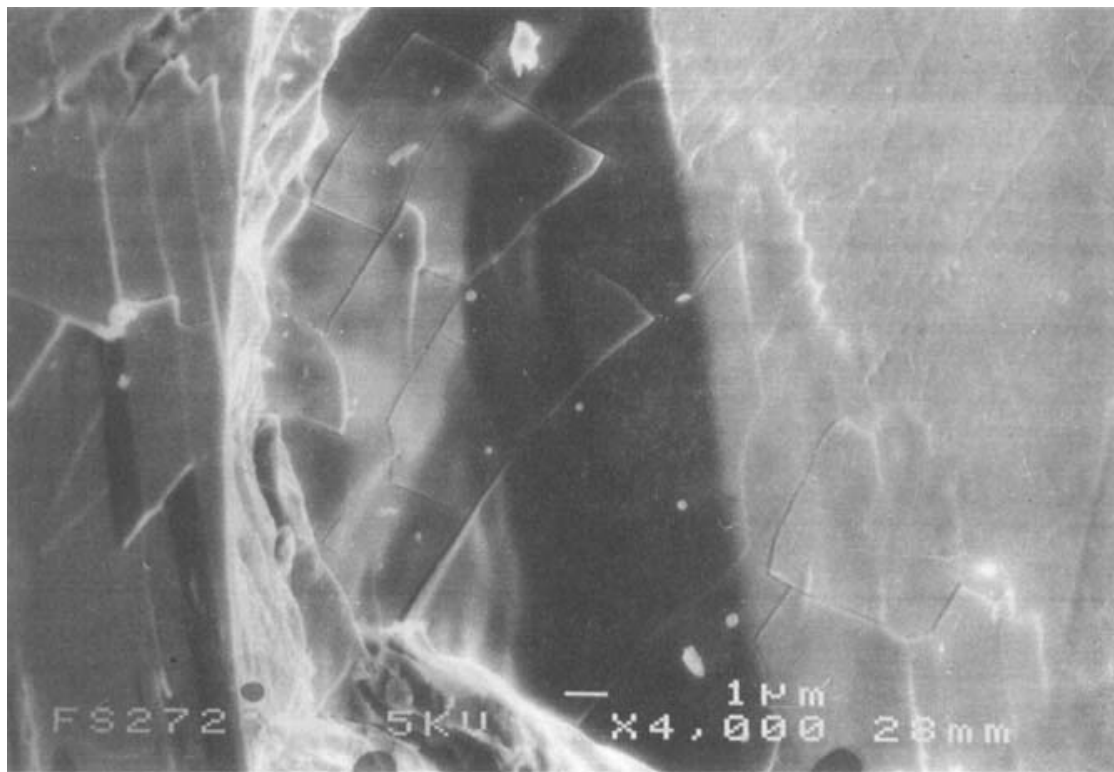

FIGURE 1(c) SE micrograph illustrating 'massive' damage at $11.7 \mathrm{~J} / \mathrm{cm}^{2}$.

electron energy below $1 \mathrm{keV}$ (see Figures 2(a) and 2(d)). This operation mode detects the positive charge built-up of partially mechanically and electrically insulated tiles forming a small chargeable capacity to ground. Furthermore, plastic deformation of a single tile near the spot centre (Figure 2(b)) and parallel sub-cracking of tiles (Figure 2(c)) are clearly discernible. Ablated tiles leave behind a substructure containing tear-off edges that are also oriented along the (111)-cleavage planes. However, no fractured surface indicates an evident topographical fine structure. This has been established for a lateral resolution of the SEM down to $1.5 \mathrm{~nm}$.

Figure 3 shows the measured tile thickness as a function of laser pulse fluence. The thickness increases monotonically with the incident fluence until about $25 \mathrm{~J} / \mathrm{cm}^{2}$. To explain this we recall that cracking occurs when the temperature rise at the surface over the whole laser spot induces shear stress exceeding the tensile strength of the material. ${ }^{4}$ Since this stress is oriented orthogonal to the surface, cracks crossing the surface at the rim of the irradiated area will fracture as seen in Figure 2(a). On the other hand, a temperature gradient into the depth can actuate fracture parallel to the (111) surface. Therefore, in combination with cross cracking near the spot periphery big tiles are formed. We assume that a threshold energy density $u_{T}=\alpha(t) F(t)$ must be deposited to cause fracture $(\alpha(z)$ is the absorption coefficient, $F(z)$ the fluence at a depth $z$ ). This is a criterion well established for calculating etch depths of polymeric materials and yields a tile thickness $t$ variation with fluence that can be described by the equation: ${ }^{5}$ $t=d \ln \alpha_{s} /\left[\left(u_{T} / F(t)\right)-\alpha_{b}\right]$.

Here, $\alpha_{s}$ is a specific surface absorption constant, attributed to light absorption by defect centres or impurities at an average depth of $d$. Since the absorption is small in a transparent material the term $(F(z)-F(0))$ can be neglected. Further, the bulk 


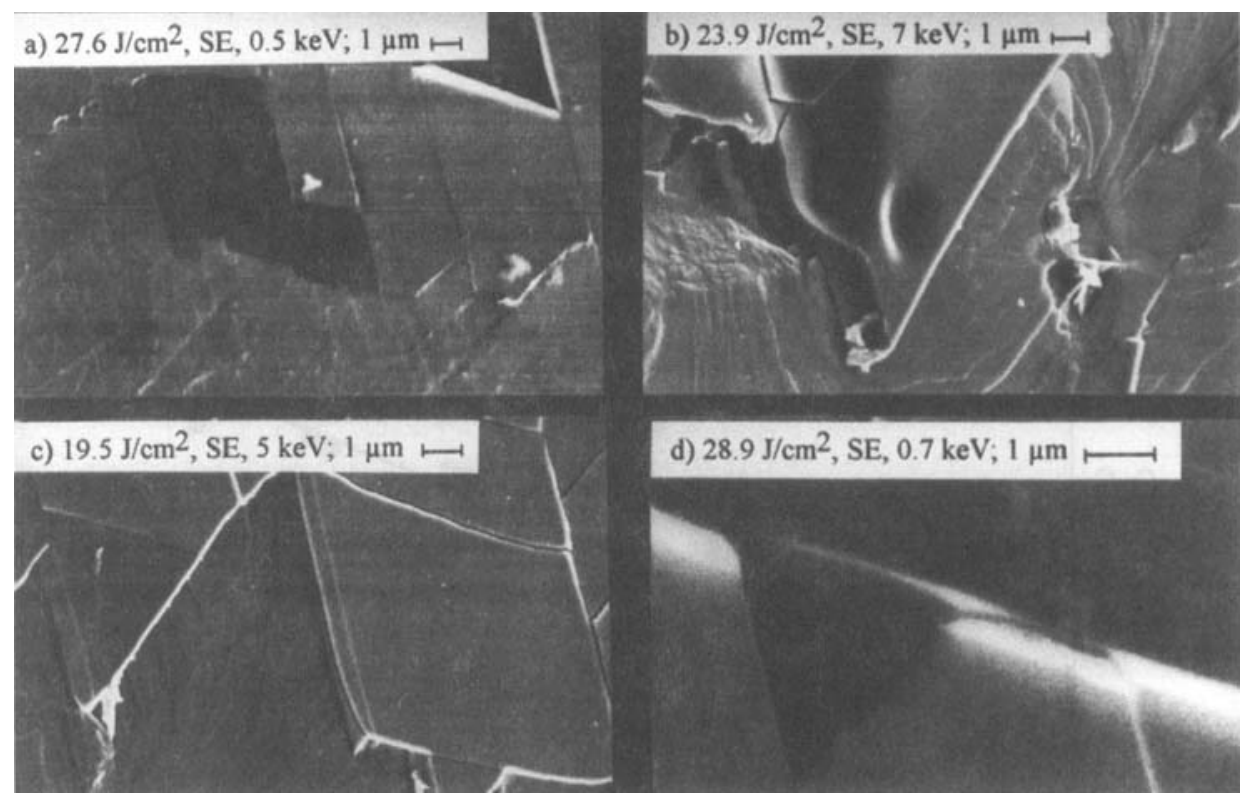

FIGURE 2 Fracture features: (a) local detachment of tiles (EBIVC), (b) surface melting near spot center (c) parallel cracking of a single tile, (d) subcracking of a small tile (EBIVC).

contribution $\alpha_{b}$ can be neglected motivated by the fact that damage occurs predominantly in the surface region. Thus we get a simplified expression for the tile thickness $t=d \ln \alpha_{s} F / u_{T}$.

This relation well fits the data as shown in Figure 3, yielding $d=0.34 \mu \mathrm{m}$ and a threshold fiuence $F_{T}=u_{T} / \alpha_{s}=1.9 \mathrm{~J} / \mathrm{cm}^{2}$. The second value is in the order of the observed threshold fluence of $4 \mathrm{~J} / \mathrm{cm}^{2}$. A theoretical value for the threshold energy density $u_{T}$ can be derived from $u_{T}=\gamma(111) / a_{\text {layer }}$ ( $\gamma$ being the surface tension) as the energy density necessary for cracking between two neighbouring $\mathrm{CaF}_{2}$ layers of spacing $a_{\text {laver }}=a / \sqrt{3}$, where $a$ is the lattice constant. Inserting numbers we obtain $u_{T}=1400$ $\mathrm{J} / \mathrm{cm}^{3}$ resulting in $\alpha_{s}=760 / \mathrm{cm}$ and a surface absorption of $\alpha_{s} d \approx 0.03$. Merely at the highest fluences above $25 \mathrm{~J} / \mathrm{cm}^{2}$ the curve does not fit the data. Presumably this indicates the onset of other damage mechanisms like dielectric breakdown.

\section{CONCLUSIONS}

Laser-induced damage of bulk $\mathrm{CaF}_{2}$ like cracking parallel to and crossing the surface plane above a threshold pulse fluence has been observed. It can be described by assuming a rather homogeneous light absorption by defects in near surface regions. Damage is caused by thermal stress under nearly homogeneous absorption across the whole spot size. The tile thickness increases monotonically with incident fluence and a simple absorption model is in good agreement with the experimental data. 


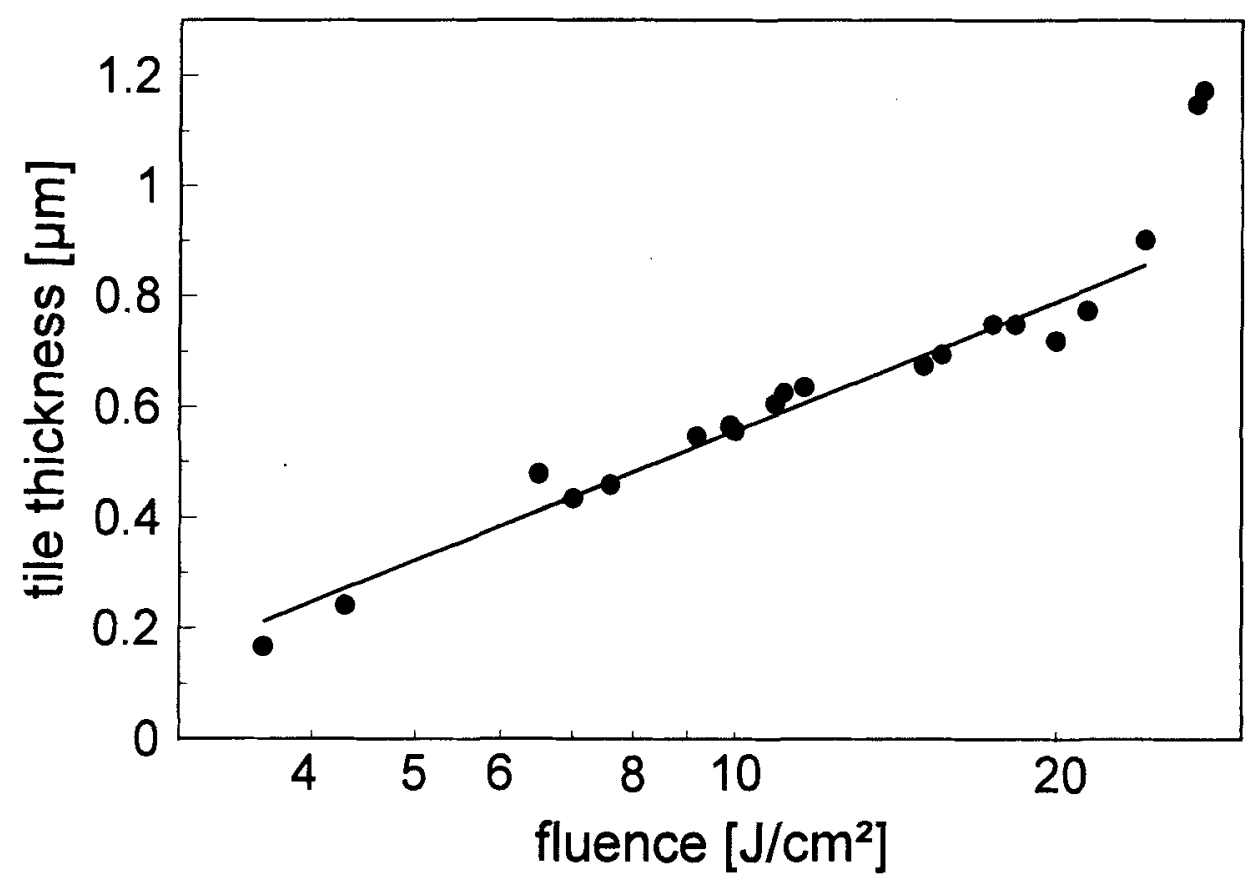

FIGURE 3 Dependence of tile thickness on laser fluence. The solid line represents a fit of the function $t=d \ln \alpha_{s} F / u_{T}$ to the data.

\section{ACKNOWLEDGEMENT}

This work was supported by Deutsche Forschungsgemeinschaft Sonderforschungsbereich 337.

\section{REFERENCES}

1. W. H. Lowdermilk and D. Milam IEEE J. Quant. Electron. QE-17 1888 (1981).

2. M. Reichling, H. Johansen, S. Gogoll, E. Stenzel, Nucl. Instr. Meth. B91, 628 (1994).

3. E. Matthias, S. Gogoll, E. Stenzel, and M. Reichling, Rad. Eff. Def. 128, 67 (1994).

4. R. L. Webb, L. C. Jensen, S. C. Langford, J. T. Dickinson, J. Appl. Phys. 74, 2323 (1993).

5. T. F. Deutsch and M. W. Geis J. Appl. Phys. 54, 7201 (1983). 